\title{
ARTículos
}

\section{Los números de fibonacci en la naturaleza y los sistemas nanoestructurados artificiales}

\author{
Clio González Zacarías, * Martha A. Palomino Ovando* \\ Gregorio H. Cocoletzi**
}

\begin{abstract}
Resumen: En este trabajo se describe y comenta la importancia y el impacto que los números de Fibonacci han tenido a lo largo de los años y su vinculación con el arte, la naturaleza y los materiales artificiales nanoestructurados, se hace una breve descripción sobre el momento histórico de su aparición, se revisan diversas formas de obtener la relación de recurrencia entre los números. Se muestran algunos ejemplos de su presencia en el arte, la naturaleza, así como resultados de propiedades ópticas de nanoestructuras de materiales artificiales construidos siguiendo esta secuencia.
\end{abstract}

\section{INTRODUCCIÓN}

\section{Historia}

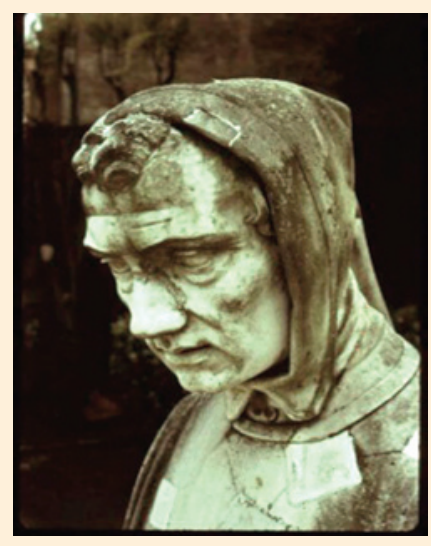

Leonardo de Pisa

A finales del siglo XII, nace en Pisa, Italia, Leonardo de Pisa (1170 a 1250), conocido como Fibonacci, apodo que le dieran de manera póstuma y que significa hijo de Bonacci. Leonardo es educado por un árabe quien lo pone en primer contacto con lo que se convertiría en una de las mayores aportaciones de los árabes al mundo occidental: nuestro actual sistema de numeración posicional.

Leonardo de Pisa, aprovechó sus viajes comerciales por todo el mediterráneo: Egipto, Siria, Sicilia y Grecia, para entablar contacto y discutir con los matemáticos más notables de la época, descubrir y estudiar a fondo los elementos de la geometría de Euclides, que tomaría como modelo de estilo y de rigor. De su deseo de poner en orden todo cuánto había aprendido de aritmética y álgebra, y de brindar a sus colegas comerciantes un potente sistema de cálculo, cuyas ventajas él había ya experimentado, escribe, en 1202, uno de los textos más famosos de la época el Liber Abaci. En este libro, mostró la importancia del nuevo sistema

\footnotetext{
* Facultad de Ciencias Físico-Matemáticas de la Benemérita Universidad Autónoma de Puebla. Av. San Claudio y Río Verde, Col. San Manuel, Ciudad Universitaria, Puebla 72570, México.

** Centro de Investigación Científica y de Educación Superior de Ensenada, Baja California, México; Centro de Nanociencia y Nanotecnología, Universidad Nacional Autónoma de México, Ensenada, Baja California; Instituto de Física, Universidad Autónoma de Puebla, México.
} 
de numeración aplicándolo a la contabilidad comercial, conversión de pesos y medidas, cálculo de intereses, cambio de moneda y otras numerosas aplicaciones[1]. En estas páginas describe el cero, la notación posicional, la descomposición en factores primos y los criterios de divisibilidad. Leonardo de Pisa brinda en su obra reglas claras para realizar operaciones con estas cifras tanto con números enteros como con fracciones; además, proporciona la regla de tres simple y compuesta, normas para calcular la raíz cuadrada de un número, así como instrucciones para resolver ecuaciones de primer grado y algunas de segundo. Sin embargo, Fibonacci es más conocido entre los matemáticos por una curiosa sucesión de números: $0 ; 1 ; 1 ; 2 ; 3$, $5 ; 8 ; 13 ; 21 ; 34 ; 55 ; 89 . .$. la que colocó en el margen de su Liber Abaci junto al conocido «problema de los conejos» que más que un problema parece un acertijo de matemáticas recreativas. El problema en lenguaje actual diría: «Suponiendo que una pareja de conejos cría otra pareja cada mes, y que los conejos son fértiles a partir del segundo mes, ¿cuántos conejos se pueden tener al cabo de un año?». En la figura 1 se muestra cómo se genera este número en las parejas de conejos a lo largo de los meses.

FIGURA 1. Crecimiento del número de parejas de conejos conforme se reproducen.

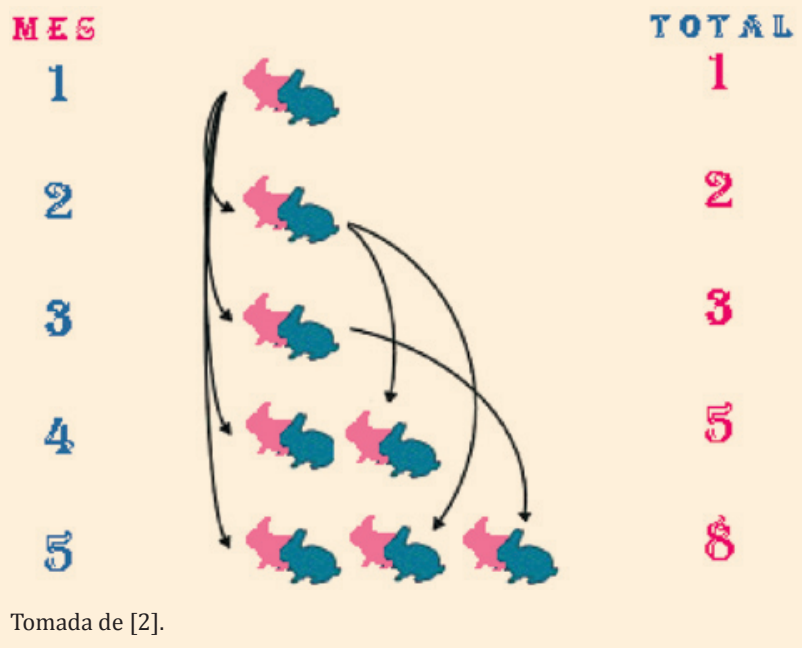

\section{RePRESEnTACIONES}

La sucesión se construye de la siguiente manera:

$$
u_{0}=0, u_{1}=1, u_{2}=1, u_{2}=2 \ldots,
$$

y la relación de recurrencia:

$$
u_{\mathrm{n}-1}+u_{n}=1, u_{\mathrm{n}+1}
$$


A la representación anterior se han hecho propuestas alternativas para generar los números de Fibonacci.

Representación algebraica

Si se desarrolla la función:

$$
f(q)=\frac{q}{1-q-q^{2}}
$$

en serie de potencias, los coeficientes de dicha expansión son los números de Fibonacci:

$$
\frac{q}{1-q-q^{2}}=0 q^{0}+1 q^{1}+1 q^{2}+2 q^{3}+3 q^{4}+5 q^{5}+8 q^{6}+13 q^{7}+\ldots
$$

\section{Fórmula de Binet}

Consideremos la sucesión de números $u_{1}, u_{2}, \ldots u_{n}$ en la cual cada término es la suma de los dos términos precedentes, es decir, para cada $n>2$ se cumple que:

$$
u_{\mathrm{n}-2}+u_{\mathrm{n}-1}=1, u_{\mathrm{n}}
$$

Observamos que para conocer todas las soluciones de ésta última ecuación basta con conocer cualesquiera dos soluciones no proporcionales de la misma (que una no sea combinación lineal de la otra) [3].

Consideremos la progresión de números $1, q, q_{2}, \ldots$

Esta progresión será una solución de la ecuación (5) si satisface la condición: $q^{n-2}$ $+q^{n-1}=q^{n}$ para toda $n$. Si dividimos entre $q^{n-2}$, la condición toma la forma $1+q=q^{2}$. Las raíces de esta ecuación cuadrática, son los números: $\alpha=\frac{1+\sqrt{5}}{2}$ y $\beta=\frac{1-\sqrt{5}}{2}$ que son las razones en las progresiones deseadas y, además, no son proporcionales entre sí. Se observa que:

$$
\alpha \cdot \beta=-1
$$

Así pues, todas las soluciones serán descritas de la forma:

$$
\mathrm{C}_{1}+\mathrm{C}_{2}, \quad \mathrm{C}_{1} \alpha+\mathrm{C}_{2} \beta, \quad \mathrm{C}_{1} \alpha^{2}+\mathrm{C}_{2} \beta^{2}, \ldots
$$

En particular, para ciertos valores de $c_{1} \mathrm{y} c_{2}$ la sucesión anterior dará la sucesión de Fibonacci. Es necesario determinar $c_{1}$ y $c_{2}$ a partir de las ecuaciones:

$$
\begin{gathered}
c_{1}+c_{2}=u_{1} \\
c_{1} \alpha+c_{2} \beta=u_{2},
\end{gathered}
$$


donde, recordamos $u_{4}=u_{2}=1$, es decir, a partir del sistema de ecuaciones:

$$
c_{1}+c_{2}=1, c_{1} \frac{1+\sqrt{5}}{2}+c_{2} \frac{1-\sqrt{5}}{2}=1
$$

Resolviendo este sistema de ecuaciones se obtiene:

$$
C_{1}=\frac{1+\sqrt{5}}{2 \sqrt{5}}, C_{2}=-\frac{1-\sqrt{5}}{2 \sqrt{5}}
$$

Que permite escribir:

$$
\mathrm{n}_{\mathrm{n}}=\mathrm{c}_{1} \alpha^{\mathrm{n}-1}+\mathrm{c}_{2} \beta^{\mathrm{n}-1}=\left(\frac{1+\sqrt{5}}{2 \sqrt{5}}\right)\left(\frac{1+\sqrt{5}}{2}\right)^{\mathrm{n}-1}-\left(\frac{1-\sqrt{5}}{2 \sqrt{5}}\right)\left(\frac{1-\sqrt{5}}{2}\right)^{\mathrm{n}-1}
$$

De donde se obtiene que:

$$
u_{n}=\frac{\left(\frac{1+\sqrt{5}}{2}\right)^{n}-\left(\frac{1-\sqrt{5}}{2}\right)^{n}}{\sqrt{5}}=\frac{\alpha^{n}-\beta^{n}}{\sqrt{5}} \quad(n=0,1,2,3, \ldots)
$$

La cual se llama fórmula de Binet, en honor al matemático que la probó por primera vez. La conexión entre la sección dorada y la serie de Fibonacci es precisamente la fórmula de Binet, donde observamos que los primeros términos son de la forma

$$
u_{0}=0, u_{1}=1, u_{2}=1, u_{2}=2, \ldots
$$

Se ha demostrado que la sección dorada es:

$$
\phi=\lim _{n \rightarrow \infty} \frac{u_{n+1}}{u_{n}}
$$

Así que considerando que para $n$ muy grande, el segundo término de la formula de Binet puede ser omitido, $\phi$ es aproximadamente igual a:

$$
\frac{1}{\sqrt{5}}\left(\frac{1+\sqrt{5}}{2}\right)^{\mathrm{n}+1} / \frac{1}{\sqrt{5}}\left(\frac{1+\sqrt{5}}{2}\right)^{\mathrm{n}}
$$

Finalmente en el límite $\phi=(1+\sqrt{5}) / 2$. 


\section{¿QUÉ ES EL NÚMERO $\phi ?$}

El número $\phi$, que de acuerdo con la expresión anterior es aproximadamente igual $1.618033988749895 \ldots$.., es un número irracional tal como lo es el número $\pi$, y cuenta con características matemáticas inusuales. La razón o proporción determinada por $\phi$ era conocida por los griegos como la "sección dorada", y por los artistas del Renacimiento, como la "proporción divina" [4]. También se le conoce como la razón dorada o la proporción áurea. Tanto $\pi$ como $\phi$ están definidos a través de una construcción geométrica. Mientras que $\pi$ es el número de veces que cabe el diámetro sobre la circunferencia, $\phi$ es la proporción de los segmentos de una línea que resultan cuando ésta se divide de tal manera que la proporción del segmento de la línea (A) respecto a la longitud del segmento de la línea (B) sea igual que la proporción de la longitud del segmento de la línea (B) a la longitud del segmento de la línea (C), como se indica en la figura 2.

FIGURA 2. Proporción de los segmentos que cumplen con $A / B=B / C$.

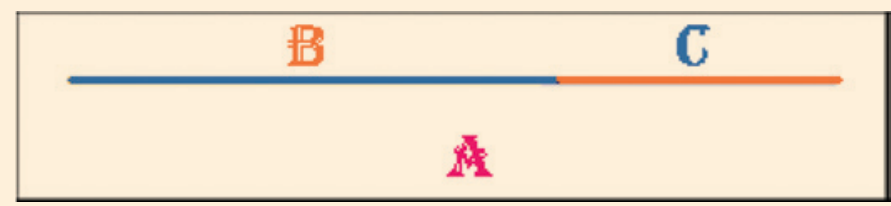

Esto significa que A es 1.618... veces B, y B es $1.618 \ldots$ veces C.

\section{El Pentágono}

Veamos cómo ésta razón está presente en otras figuras. Tomemos un pentágono con cinco lados iguales y conectemos todos sus puntos para formar una estrella de cinco puntas. Las razones de la longitud de los segmentos de línea resultantes están todos basados en $\phi$. En la imagen, notamos que:

FIGURA 3. Pentágono.

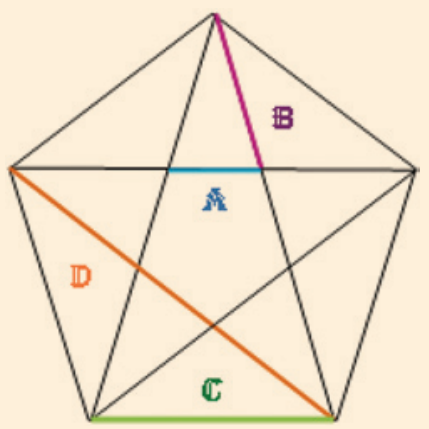




\section{Espiral áurea (espiral dorada)}

Si sumamos los cuadrados de cualquier serie de los números de Fibonacci, van a igualar el último número de Fibonacci usado en la serie por el siguiente número de Fibonacci. Esta propiedad se ve en la espiral dorada, que se encuentra en la concha del molusco Nautilus así como en las galaxias [5]. Expresado en forma matemática se tiene:

$$
1^{2}+1^{2}+2^{2}+3^{2}+5^{2}+\ldots+[F(n)]^{2}=F(n) \times F(n+1)
$$

FIGURA 4. Se ilustra la forma en que se construye la espiral. Obsérvese cómo los lados de los cuadrados siguen la secuencia de Fibonacci.
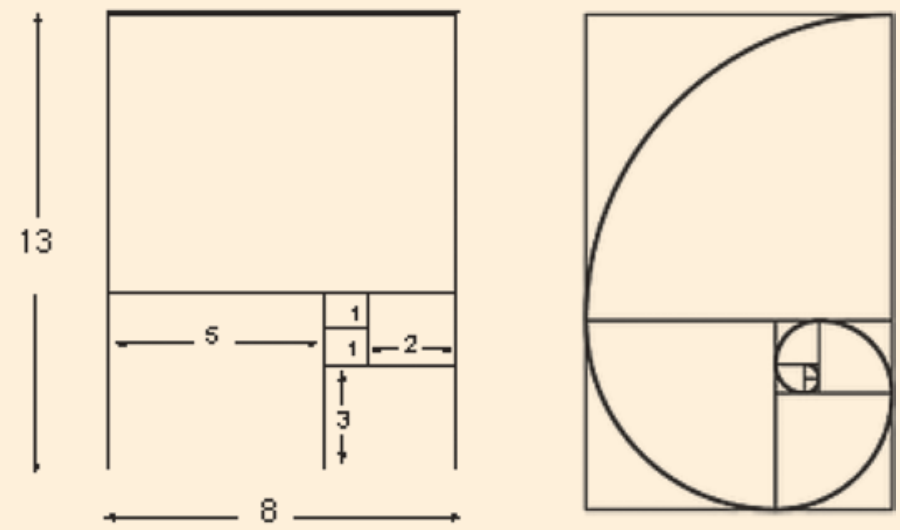

Por ejemplo si $n=5$ tendremos $1^{2}+1^{2}+2^{2}+3^{2}+5^{2}=5 \times 8$. Obsérvese en la figura 4 la construcción geométrica de los cuadrados de los primeros números de Fibonacci. Si se extiende para un número mayor de números de Fibonacci obtenemos una espiral, que se construye siguiendo la serie y conectando las esquinas opuestas de los cuadrados de medida igual a cada elemento de la serie.

FIGURA 5. Presencia en la naturaleza de la espiral: galaxias, algunos tipos de caracol, huracanes, etc. Fotos tomadas de $[5,6]$.
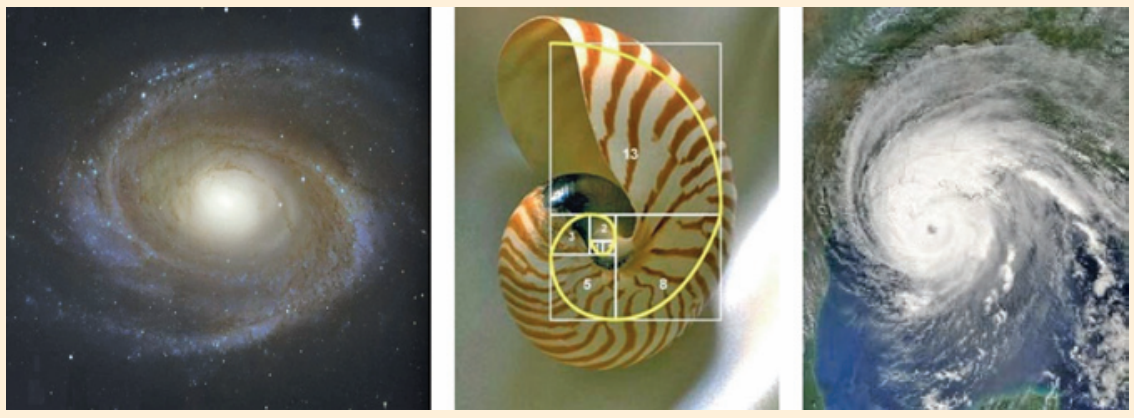


\section{Los números de Fibonacci en la naturaleza}

Hay muchos elementos relacionados con la sección áurea en la naturaleza. A continuación damos una lista de ejemplos donde los números de Fibinacci están presentes:

- La relación entre la cantidad de abejas macho y abejas hembra en un panal.

- La relación entre la distancia entre las espiras del interior de cualquier caracol.

- La relación entre los lados de un pentáculo.

- La disposición de los pétalos de las flores (el papel del número áureo en la botánica recibe el nombre de Ley de Ludwig).

- La distribución de las hojas en un tallo.

- La relación entre las nervaduras de las hojas de los árboles.

- La relación entre el grosor de las ramas principales y el tronco, o entre las ramas principales y las secundarias.

- La distancia entre las espirales de una piña.

- La anatomía de los humanos se basa en una relación $\phi$ exacta, esto corresponde a: a) la relación entre la altura del humano y la altura de su ombligo; b) la distancia del hombro a los dedos y la distancia del codo a los dedos; c) la distancia entre la altura de la cadera y la altura de la rodilla; $d$ ) la distancia del primer hueso de los dedos (metacarpiano) y la primera falange, o entre la primera y la segunda, o entre la segunda y la tercera, en todos los casos el cociente es $\phi ; e$ ) la relación entre el diámetro de la boca y el de la nariz; $f$ ) el diámetro externo de los ojos y la línea interpupilar; g) cuando la tráquea se divide en sus bronquios, si se mide el diámetro de los bronquios por el de la tráquea se obtiene $\phi$. Está comprobado que a mayor cantidad de estas relaciones en el cuerpo y en el rostro hacen que la mayoría de las personas reconozcan a esos individuos como lindos, bellos y proporcionados.

FIGURA 6. Presencia en la naturaleza de la secuencia de Fibonacci. Fotos tomadas de [7].

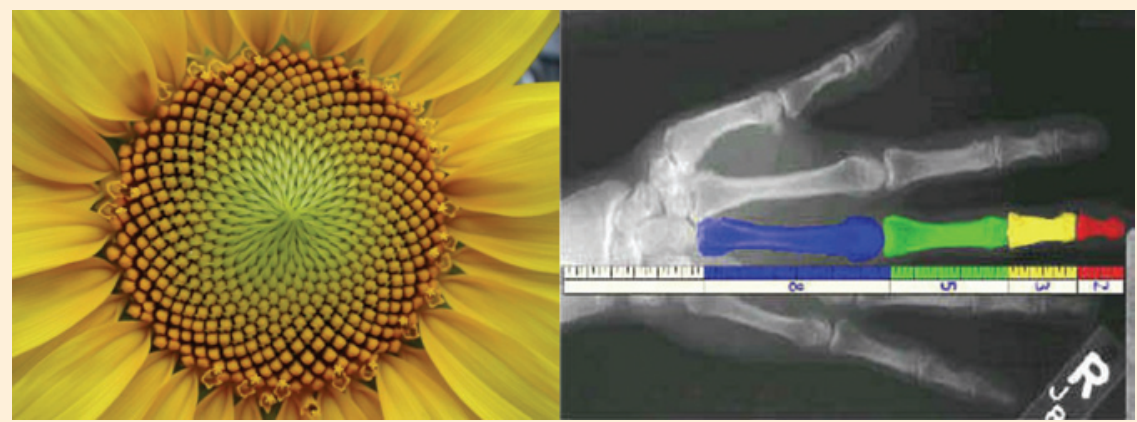

La sección áurea en el arte

De manera similar a la presencia de los números de Fibonacci en la naturaleza, la sección áurea está presente en el arte. Aquí mencionamos algunos ejemplos: 
- La relación entre las partes, el techo y las columnas del Partenón, en Atenas.

- En los violines, la ubicación de las efes (los "oídos", u orificios en la tapa) se relacionan con el número áureo.

- El compositor mexicano Silvestre Revueltas (1899-1945) utilizó también el número áureo en su obra Alcancías, para organizar las partes.

- En las relaciones entre altura y ancho de los objetos y personas que aparecen en las obras de Miguel Ángel, Alberto Durero y Leonardo Da Vinci, entre otros.

- Las relaciones entre articulaciones en el hombre de Vitruvio y en otras obras de Leonardo da Vinci.

- En las estructuras formales de las sonatas de Wolfgang Amadeus Mozart, en la Quinta Sinfonía de Beethoven, en obras de Franz Schubert y Claude Debussy.

- En el Arte Póvera, movimiento artístico italiano de los años 1960, muchas de sus obras se basan en esta sucesión.

FIGURA 7. El Partenón griego fue construido también respetando las proporciones áurea, al igual que el rostro de la Gioconda, proporcionado con rectángulos áureos. Fotos tomadas de [8, 9].
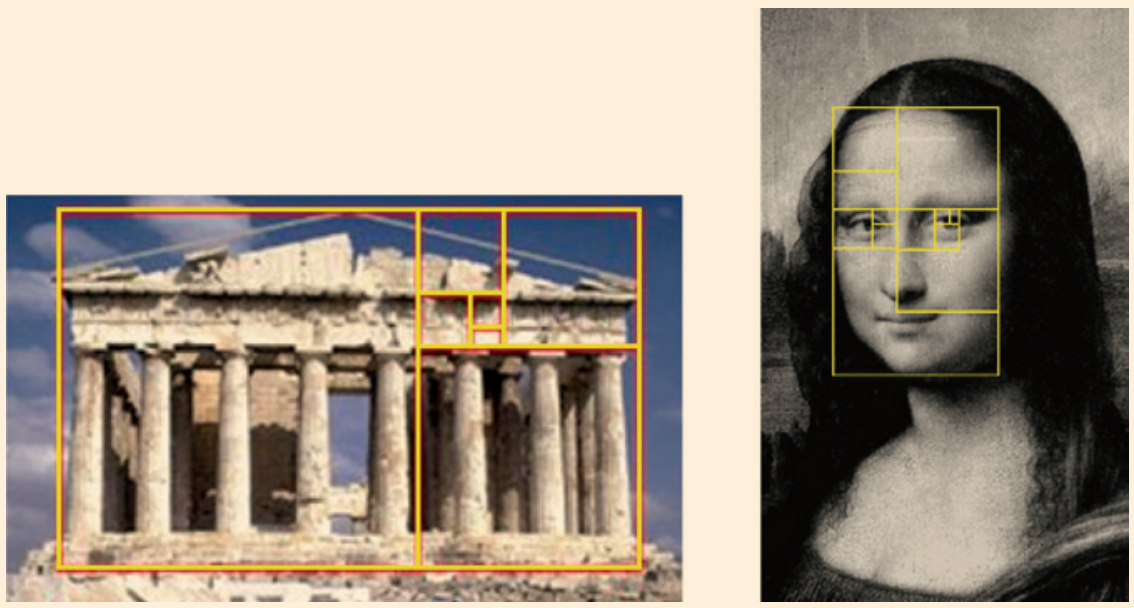

Hoy en día la sección áurea se puede ver en multitud de diseños. El más conocido y difundido sería la medida de las tarjetas de crédito, la cual también sigue dicho patrón, así como también en las cajetillas de cigarrillos.

\section{LOS NÚMEROS DE FIBONACCI EN LA CONSTRUCCIÓN DE NANOESTRUCTURAS}

Con los avances tecnológicos de los últimos años se ha logrado la fabricación de nanoestructuras artificiales con propiedades físicas propicias para sus aplicaciones en la industria. Así, las técnicas de crecimiento epitaxial de nano-heteroestructuras se han empleado para fabricar sistemas multicapas periódicas y aperiódicas con aplicaciones en la industria optoelectrónica. Esto se ha motivado por la búsqueda de nuevos materiales con propiedades físicas específicas para aplicaciones tecnológicas. 
Una estructura periódica es aquella en la que una unidad se repite en la estructura del sistema. Por otro lado, un sistema aperiódico se identifica como aquel que no tiene periodo, éste se puede formar, por ejemplo, con la secuencia de los números de Fibonacci. Lo anterior se detalla más adelante.

En la década de los años setenta del siglo pasado, Tsu y Esaki [10] estudiaron las propiedades de transporte de nanoestructuras formadas de capas delgadas; al sistema se le conoce con el nombre de superred. Esta superred se forma de capas alternadas de dos materiales diferentes. En este sistema una bicapa forma la celda unitaria que se repite en la estructura. Posteriormente, en 1985, R. Merlin y colaboradores [11], mediante el uso de la epitaxia de haces moleculares (MBE), crecieron heteroestructuras con la secuencia de los números de Fibonacci. La caracterización mediante rayos X y Raman de estas estructuras mostraron propiedades físicas interesantes. Debido a las posibles aplicaciones tecnológicas, las estructuras construidas con la secuencia de Fibonacci han recibido considerable atención de la comunidad científica. A la fecha, se han estudiado tanto propiedades ópticas como de transporte en sistemas de Fibonacci. En este reporte queremos mostrar un ejemplo de las propiedades ópticas de sistemas que se construyen siguiendo la secuencia de los números de Fibonacci.

En la actualidad, existe mucho interés en estudiar la propagación de la radiación electromagnética en estructuras fotónicas. Esto se ha motivado por las aplicaciones en la industria optoelectrónica. Una estructura fotónica o cristal fotónico puede ser de uno, dos o de tres dimensiones, y se puede ordenar con o sin periodicidad. Aquí nos restringiremos a estudiar sistemas en una dimensión. Un cristal fotónico unidimensional se puede construir alternando dos capas delgadas de dos materiales diferentes, es decir, dos capas con índice de refracción $n_{1}$ y $n_{2}$, respectivamente. Una de las propiedades físicas importantes que se investigan en los cristales fotónicos es la estructura de bandas, que describe el rango de frecuencias en que a la luz se le "permite" (bandas) o se le "prohíbe" (brechas) propagar a través del cristal. La aparición de bandas y brechas para la propagación de ondas electromagnéticas es una consecuencia de la periodicidad de la estructura. Esta propiedad se puede usar para la fabricación de filtros para la propagación direccional de la luz. Tomando en cuenta esta situación se puede explicar por qué en un sistema aperiódico la propagación se frustra, dando como consecuencia un sistema que no propaga radiación electromagnética. Por otro lado, se puede construir una estructura cuasi-periódica, por ejemplo, tomando como base las estructuras construidas con los números de Fibonacci. Así, se puede tomar como la unidad cualquier estructura de Fibonacci y repetirla de manera indefinida, como se explica más adelante. Como ejemplo ilustrativo en este trabajo presentamos las bandas y brechas que se obtienen en estructuras cuasiperiódicas generadas con los números de Fibonacci.

En 1967, Victor Veselago [12] propuso las consecuencias de un material hipotético con refracción negativa. En este material hipotético, contrario a lo observado en todos los materiales existentes hasta esa época, se preguntaba si la asimetría sería posible de alcanzar aunque fuese en un material artificial, y concluyó que de existir tales materiales exhibirían características notables que modificarían todos los fenómenos electromagnéticos observados hasta la fecha. Materiales con índice de refracción negativo no se encuentran disponibles en la naturaleza, pero éstos se pueden construir. Para tener un material de índice de refracción negativo se requiere que tanto la permitividad eléctrica como la permeabilidad magnética sean negativos, esto da lugar 
a un índice de refracción negativo. Los materiales con índice de refracción negativo también se les llaman metamateriales. Sus posibles aplicaciones son variadas, entre los que se pueden nombrar los superlentes y el efecto de la invisibilidad, entre otros.

A continuación trataremos de explicar la refracción de la luz en materiales con índice de refracción negativo. Para ello consideremos la figura 8. El rayo incidente y el refractado se indican con la línea roja y la normal a la interface de separación entre los medios con una línea negra. La parte de la izquierda muestra la refracción negativa y la parte de la derecha presenta la refracción positiva. Puede apreciarse que la dirección del haz refractado tiene diferentes direcciones dependiendo de si la refracción es positiva o negativa. Para precisar, un material con índice de refracción negativa induce una refracción negativa.

FIGURA 8. En esta figura se representa esquemáticamente la refracción negativa y positiva de la luz. Imagen tomada de [13].

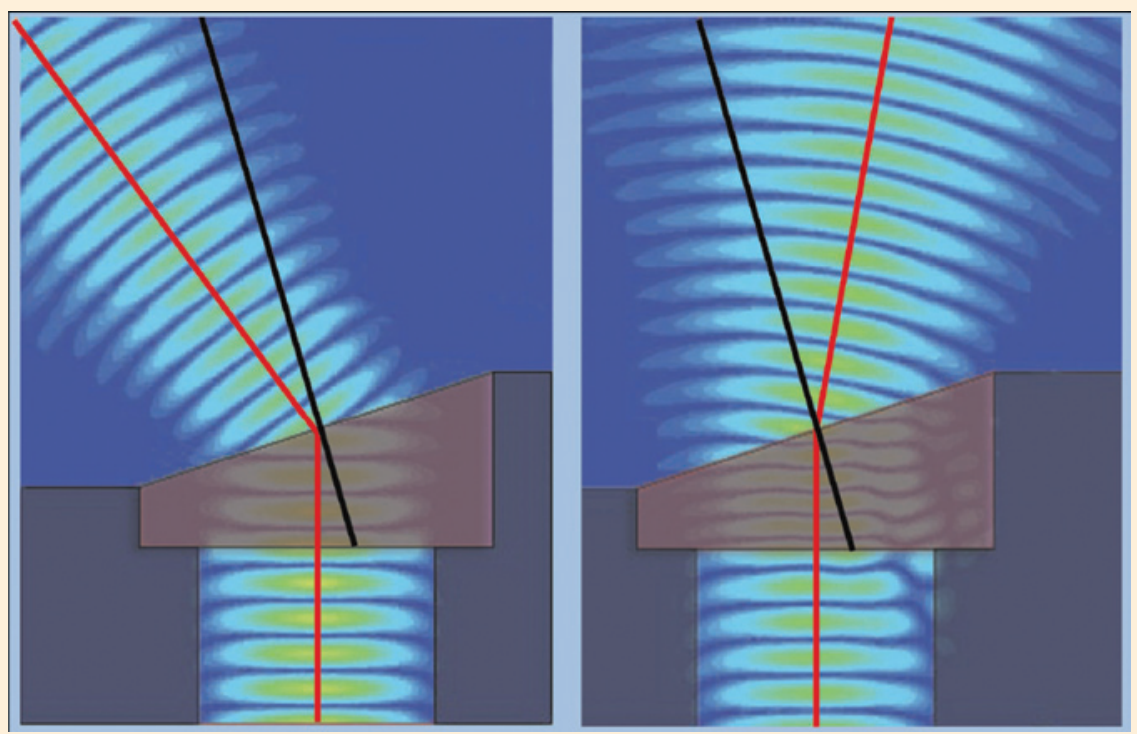

\section{ESTRUCTURA DE BANDAS DE UNA RED DE FibONACCI}

La secuencia de Fibonacci nos permite estructurar cristales que no son periódicos, pero gracias a que llevan una secuencia ordenada y no se generan de manera aleatoria tampoco son amorfos; a este tipo de estructuras intermedias se les denomina cuasicristales. Como ya se mencionó anteriormente, nos restringiremos a estudiar sólo sistemas unidimensionales. Consideremos la estructura de Fibonacci que se construye a partir de dos bloques A y B que siguen la regla de generación de los números de Fibonacci, ambos tienen los mismos componentes pero sus dimensiones (ancho de las capas) son diferentes. Así que si $S_{0}=\{A\}$ y $S_{1}=\{A B\}$ usando la secuencia de Fibonacci se puede construir $S_{2}=\left\{S_{1} S_{0}\right\}=\{A B A\}, S_{3}=\left\{S_{2} S_{1}\right\}=\{A B A A B\}$, y de manera gene$\operatorname{ral} S_{j}=\left\{S_{j-1} S_{j-2}\right\}=\{A B A\}$. En este caso hemos elegido como componente de cada bloque 
A y B dos materiales uno de ellos metamaterial, y otro dieléctrico (por comodidad se escoge aire).

Cuando el índice de refracción (m) es negativo $n<0$ la estructura de bandas tiene diferentes comportamientos en comparación a cuando es positivo. Para describir la propagación de las ondas electromagnéticas en estructuras multicapas, primero definimos el sistema de coordenadas. Las interfases son paralelas al plano $x y$, la propagación de las ondas sucede en el plano $x z$. En cada capa se satisface la relación de dispersión $\omega=\omega(\mathrm{k})=\mathrm{kc} / \mathrm{n}$, la frecuencia angular de la onda es una función del vector de propagación $k$. En esta expresión el índice de refracción se define como $\mathrm{n}=\sqrt{\varepsilon \mu}$, donde $\varepsilon$ es la permitividad eléctrica, $\mu$ la permeabilidad magnética y $c$ la velocidad de la luz en el vacío. Si uno de los medios tiene $n<0$, entonces:

$$
\omega=\frac{\mathrm{c} \sqrt{\mathrm{k}_{\mathrm{z}}^{2}+\beta^{2}}}{-\sqrt{\varepsilon_{\mathrm{j}} \mu_{\mathrm{j}}}} .
$$

En general $k_{z j}=k_{z j R}+j k_{z j l}$ es compleja . Para incidencia normal de la onda $\beta \neq 0$, entonces,

$$
\omega=\frac{\mathrm{c} \sqrt{\mathrm{k}_{\mathrm{z}}^{2}}}{-\sqrt{\varepsilon_{\mathrm{j}} \mu_{\mathrm{j}}}}
$$

y $\omega$ sería una cantidad compleja, lo cual no es aceptable físicamente. A estos modos no accesibles se les denominan modos espurios. Si $\beta \neq 0$ (incidencia oblicua) puede ocurrir que $k_{z j R}=0$ y $k_{z j l}^{2}<\beta^{2}$, esto proporciona una $\omega$ real, por lo tanto, son modos posibles de propagación y se le denomina tunelamiento fotónico [14].

A continuación damos ejemplos específicos de las propiedades ópticas de cristales fotónicos unidimensionales. Para ello se han realizado cálculos de la estructura de bandas y de la respuesta óptica en estructuras periódicas (superredes). Se hicieron proyecciones de las estructuras de bandas, para una red periódica y una de Fibonacci. En la figura 9 se muestran gráficas de la estructura de bandas (lado izquierdo) y de patrones de transmisión de las ondas electromagnéticas. En la figura 9a se reportan los resultados para la red periódica. La sección sombreada de la izquierda muestra las bandas permitidas (sección gris) y brechas prohibidas (sección en blanco), mientras que en verde se indican las frecuencias de tunelamiento. Las curvas representan la amplitud de transmisión para dos geometrías, una tiene periodo 5 capas y otra tiene periodo 10 capas. Los picos de la transmisión están en frecuencias de la banda y corresponden a resonancias. En la zona prohibida el tunelamiento se anula indicando que no hay propagación de ondas. Por otro lado, a frecuencias mayores a la zona prohibida aparecen una serie de picos que representan el tunelamiento de las ondas.

En la figura 9b se presentan las bandas y el espectro de tunelamiento para la estructura geométrica generada con los números de Fibonacci, en el ejemplo $\mathrm{N}_{\mathrm{F}}=3$. Las regiones de frecuencias sombreadas en gris representan las bandas permitidas, la región en blanco es para la brecha prohibida y la parte de color verde es para los modos de tunelamiento. Los picos del tunelamiento representan múltiples resonancias de las ondas en las capas que forman la estructura. Los parámetros usados en este 
cálculo corresponden a una permitividad eléctrica y permeabilidada magnética dadas por [15]:

$$
\varepsilon(\omega)=\frac{\left(\omega^{2}-\omega_{\mathrm{p}}^{2}\right)}{\omega^{2}}, \quad \mu(\omega)=\frac{\left(\omega^{2}-\omega_{\mathrm{b}}^{2}\right)}{\left(\omega^{2}-\omega_{0}^{2}\right)}
$$

donde $\omega_{p, b, o}=2 \pi f_{p, b, o}$ con $f_{p}=12 \mathrm{GHz}, f_{b}=6 \mathrm{GHz}$ y $f_{o}=4 \mathrm{GHz}$. El medio dieléctrico (RHM) para intercalar con el material izquierdo (LHM) es el aire. Los espesores son de $d_{1}=d_{2}$ $=0.5 \mathrm{~cm}$. Y las gráficas de transmisión fueron calculadas para un ángulo de $35^{\circ}$.

FIGURA 9. Estructura de bandas y espectros de transmisión para dos casos: a) estructura periódica; b) estructura de Fibonacci.

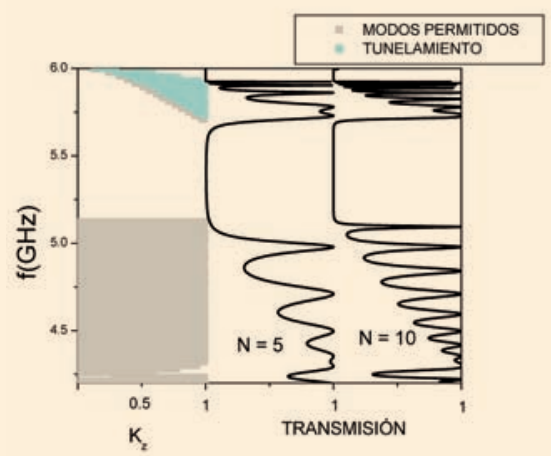

a)

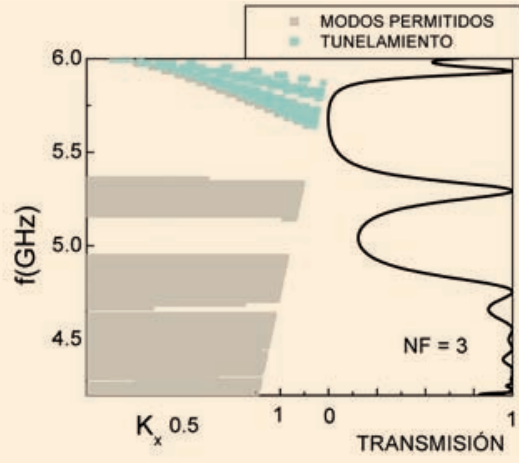

b)

Otro ejemplo se ilustra en la figura 10. La gráfica muestra la amplitud de tunelamiento en estructuras que se construyeron con $\mathrm{N}_{\mathrm{F}}=3,4$ y 6. Similar a la figura 9, los espectros despliegan las bandas permitidas, así como las brechas prohibidas. Puede apreciarse que en las bandas existe estructura que se genera por las múltiples resonancias de Fabry-Perot, que aumentan con el número de Fibonacci y con el ancho de las capas.

Las estructuras laminadas que hemos considerado aquí tienen anchos más grandes que los nanométricos; sin embargo, es posible construir estructuras nanométricas cuyas respuestas ópticas y de transporte muestren propiedades interesantes y útiles para las aplicaciones tecnológicas. Cabe mencionar que existen estudios de las propiedades de transporte electrónico, donde la probabilidad de tunelamiento presente el fenómeno de autosimilaridad [16 - 18]. 
FIGURA 10. Coeficientes de transmisión considerando como celda unitaria los bloques con $\mathrm{N}_{\mathrm{F}}=3,4$ y 6 .

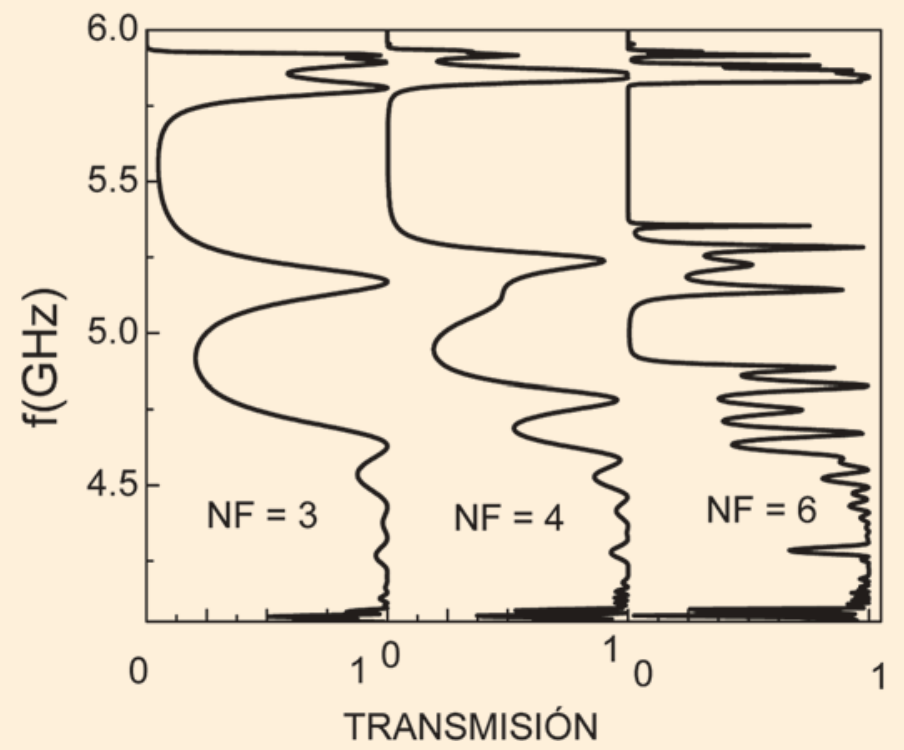

\section{REFERENCIAS}

[1] H. E. Huntley. 1970. The divine proportion: A study in mathematical Beauty. Dover Publications, Inc.

[2] <http://www.portaleureka.com/accesible/matematicas/103-matematicas/117-fibonacci-y-el-numero-de-oro>.

[3] N. N. Vorobyov. 1973. Los números de Fibonacci. México; Limusa.

[4] R. Knott, D.A.Quinney. The life and numbers of Fibonacci. +Plus Magazine. (c) 1997-2009, Millennium Mathematics Project, University of Cambridge. September 1997

[5] "The Fibonacci Numbers". Time Magazine, in partnership with CNN Friday, abril 4, 1969

[6] <http://concepcionabraira.wikispaces.com/La+secci\%C3\%B3n+\%C3\%A1urea>.

[7] <http://www.environmentalgraffiti.com/featured/fibonacci-sequence-illustratednature/ 10867>.

[8] <http://britton.disted.camosun.bc.ca/goldslide/jbgoldslide.htm>.

[9] <http://sobrecuriosidades.com/2009/05/07/secuencia-de-fibonacci-y-divina-proporcion-matematicas-que-rigen-la-vida/>.

[10] P. A. Lebwohl and R. Tsu, J. App. Phys. 41, 2664 (1970); R. Tsu and L. Esaki, Applied Physics Letters 19, 246 (1971); R. Tsu and L. Esaki, Tunneling in a finite superlattice, Applied Physics Letters 22, 562 (1973).

[11] R. Merlin, K. Bajema, R. Clarke, F.-Y. Juang, and P. K. Bhattacharya. 1985. “Quasiperiodic GaAs-AlAs heterostructures", Physical Review Letters, 55, 1768 (1985).

[12] V. G. Veselago. 1968. Electrodynamics of substances with simultaneously negative values of e and m. Sov. Phys. Usp. 10,509 (1968). 
[13] J. B. Pendry, D. Smith. 2004. "Reversing light with negative refraction". Physics Today 37. junio.

[14] Liang Wu; Sailing He; Linfang Shen. 2003. "Band structure for a one-dimensional photonic crystal containing left-handed materials". Phys. Rev. B 67: 235103

[15] Dutta Gupta, S.; Arun, R.; Agarwal, G. S. 2004. "Subluminal to superluminal propagation in a left-handed medium". Phys. Rev. B 69: 113104.

[16] Luca Dal Negro, Claudio J. Oton,1 Zeno Gaburro, Lorenzo Pavesi, Patrick Johnson,2 Ad Lagendijk, 2003. "Light Transport through the Band-Edge States of Fibonacci Quasicrystals", Phys Rev. Lett. 90, 055501.

[17] Mher Ghulinyan, Claudio J. Oton, Luca Dal Negro y Lorenzo Pavesi. 2005. “Light-pulse propagation in Fibonacci quasicrystals", Phys. Rev. B 71, 094204.

[18] M. Palomino-Ovando, Gregorio H. Cocoletzi, y C. Pérez-López, Phys. Lett. A 213, 191 (1996); M. Palomino y G. H. Cocoletzi. 1998. Superlattices and Microstructures 24, 3. 